Christopher B. Hays

\title{
Death in the Iron Age II and in First Isaiah
}

[Tod in der Eisenzeit II und im Jesajabuch.]

Veröffentlicht auf Englisch.

Der Tod ist eines der Hauptthemen in dem Buch Jesaja, obwohl er als solcher bisher nicht allgemein wahrgenommen wurde.

CHRISTOPHER B. HAYS

Death in the Iron Age II and in First Isaiah Christopher B. Hays zeigt, was über den Tod im Alten Orient während der Eisenzeit II bekannt war. Dabei berücksichtigt er die Vorstellungen und Bräuche in Mesopotamien, Ägypten, Syrien-Palästina und Judäa/Israel. Indem er sowohl Textinformationen als auch archäologische Gegebenheiten in seine Untersuchung einbezieht, kann der Autor einen Überblick und eine Analyse der bestehenden wissenschaftlichen Literatur über diese Themen aus vielerlei Perspektiven geben. Er konzentriert sich auf die Bedeutung des Textes für seine Autoren als auch für seine ersten Zuhörer, beschreibt, wie die 'Rhetorik des Todes' in ihrem historischen Kontext funktionierte und bietet somit neue Interpretationen von mehr als einem Dutzend Textstellen in Jesaja 5-38. Er zeigt, wie diese die Bildlichkeit des Todes, die Teil ihres kulturellen Kontextes war, benutzten und wie damit neue Wege beschritten wurden.

Christopher B. Hays Born 1973; 2008 Ph.D. at Emory University; presently D. Wilson Moore Assistant Professor of Ancient Near Eastern Studies at Fuller Theological Seminary in Pasadena, CA.

2011. XVIII, 445 Seiten. FAT 79

SBN 978-3-16-151751-8

DOI 10.1628/978-3-16-151751-8

eBook PDF 159,00€

ISBN 978-3-16-150785-4

Leinen $159,00 €$

Jetzt bestellen:

https://mohrsiebeck.com/buch/death-in-the-iron-age-ii-and-in-first-isaiah-9783161517518?no cache=1

order@mohrsiebeck.com

Telefon: +49 (0)7071-923-17

Telefax: $+49(0) 7071-51104$ 